\title{
Estimaciones de la población desde la tierra como desde el cielo: el caso de Tenosique
}

\section{Estimates of the population from the earth as from the sky: the case of Tenosique}

\author{
Manuel Ordorica*, José Luis Silvan** \\ y Juan Manuel Núñez** \\ * El Colegio de México, México \\ ** CentroGeo de CONACYT, México
}

\section{Resumen}

El objetivo del artículo es realizar estimaciones de población de Tenosique, en el estado de Tabasco, con el propósito de probar una metodología que podría ser aplicable para hacer estimaciones de población de áreas pequeñas. Esta técnica combina censos, conteos y encuestas con imágenes satelitales para hacer estimaciones de población. Se eligió Tenosique para estudiar el efecto del crecimiento de la población sobre la zona de influencia del rio Usumacinta, derivado de la importante población flotante de migrantes centroamericanos. El método que se propone utiliza información continua en intervalos pequeños de tiempo real, pero desde el espacio, ya que el satélite pasa de manera frecuente por el mismo lugar. El método consiste en establecer una relación entre el crecimiento del área y el aumento de la población. La técnica usada es el Filtro de Kalman.

Palabras clave: Población de áreas pequeñas, Filtro de Kalman, imágenes satelitales, demografía matemática, ciudad de Tenosique.

\section{Abstract}

The objective of the article is to realize estimates of population of Tenosique, in the state of Tabasco, with the purpose of testing a methodology that could be applicable to make estimations of population of small areas. This technique combines censuses, counts and surveys with satellite images to make population estimates. Tenosique was chosen to study the effect of population growth on the zone of influence of the Usumacinta River, derived from the important floating population of Central American migrants. The proposed method uses continuous information in small, real-time intervals, but from space, as the satellite frequently passes through the same location. The method is to establish a relationship between the growth of the area and the increase of the population. The technique used is the Kalman filter.

Keywords: Population of small areas, Kalman filter, satellite images, mathematical demography, city of Tenosique. 


\section{INTRODUCCIÓN}

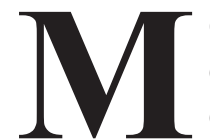

éxico tiene una larga historia en el levantamiento de censos de población, en registros administrativos y en la realización de encuestas. Además, desde la mitad del siglo pasado cuenta con proyecciones de población a nivel nacional. Las primeras perspectivas demográficas para México las realizó Naciones Unidas y se publicaron en 1954. Nuestro interés por aproximarnos al futuro es reciente. Apenas un suspiro desde que los primeros pobladores habitaran nuestro territorio. Parecería que hasta ahora nos interesa nuestro porvenir y que en el pasado no teníamos tiempo de ver más que el presente o reflexionar acerca de nuestra historia.

A lo largo de varios años desde que México naciera como país independiente, el Estado Mexicano se ha preocupado por tener un gran acervo de información sociodemográfica de uso común. Además, ha hecho un gran esfuerzo por tener importantes instituciones generadoras de datos, de ahí el nacimiento del hoy Instituto Nacional de Estadística y Geografía (INEGI) e instituciones de investigación como El Colegio de México que han formado especialistas para estudiar los hechos sociodemográficos. Muchos de ellos son científicos con experiencia, capaces de leer y entender la información, y traducirla para la elaboración de políticas públicas. También hace poco más de 40 años se creó el Consejo Nacional de Población (CONAPO), institución encargada de elaborar los diagnósticos y las proyecciones oficiales de población. En nuestro país se ha producido mucha información, tanta que la generación de datos le ha ganado a la etapa de producción de estudios derivados de los datos. No se trata de tener un inmenso cúmulo de datos sin analizar, sino algunos seleccionados con puntería que nos permitan modificar una posible trayectoria negativa de nuestra vida como seres humanos que residimos en este inmenso territorio.

Con el paso del tiempo se han incrementado las necesidades de datos desglosados a pequeños niveles de análisis a fin de ejecutar programas de desarrollo, debido a que cada vez se le dan mayores responsabilidades a los gobiernos municipales en la toma de decisiones. Además, es ahí donde habitan generalmente grupos indígenas. Ya resolvimos lo que es posible a nivel del país, ahora resulta que necesitamos datos a nivel micro-regional. Esto implica por un lado, tener información a esos niveles, y por la otra, tener personal en esos lugares cada vez más preparado que pueda analizar 
los datos para realizar acciones efectivas. A este país le faltan especialistas en el campo del análisis de cifras. No hemos preparado lo suficiente a las diferentes generaciones, todo lo queremos resolver con palabras y discursos. Un problema que se presenta en la producción de datos demográficos y sus proyecciones ocurre cuando las unidades de análisis son pequeñas, como por ejemplo, a nivel de municipio o de localidad.

Los resultados están sujetos a una gran variabilidad. Son datos muy volátiles por que la movilidad de la población es muy intensa y fluctúa mucho. Por eso las proyecciones de población de México se han realizado principalmente a nivel nacional y por entidad federativa. El seguimiento y evaluación de las perspectivas demográficas en nuestro país se hace sólo de las que se realizan a nivel nacional. Las proyecciones de población estatales no se evalúan, sólo se utilizan en forma mecánica por los tomadores de decisión en los estados como datos curiosos o para fines de asignación del presupuesto. Se toman como verdades absolutas; hace falta una mente más analítica. Lo más certero son nuestros censos, son nuestra mejor verdad. En México nos hace falta saber qué hacer con las proyecciones. En el pasado, las proyecciones que se hicieron a nivel nacional señalaban que México tendría 151 millones de personas en el año 2000. Sirvieron para cambiar la Política de Población a fin de frenar el ritmo de crecimiento demográfico. Hay que plantear proyecciones tendenciales y otras programáticas que nos indiquen hacia dónde movernos. Las proyecciones se hacen para que no se cumplan.

En la planeación cada vez más se requiere de proyecciones de población de áreas pequeñas, pues son lugares habitados en muchas zonas por pueblos indígenas. Ellos viven en zonas aisladas y dispersas, cuidando la naturaleza de sus territorios. El interés por conocer el número de habitantes tiene que ver con el hecho de que hay pueblos en los que se está extinguiendo la población y con ellos sus lenguas que son una joya de la cultura mexicana. Quieren vivir aislados porque quieren mantener su cultura y su historia, hecho poco comprendido por las generaciones modernas. Por otro lado, es importante conocer el impacto de la dinámica demográfica sobre el medio ambiente a niveles micro-regionales. ¿Será que son los indígenas los que mejor cuidan nuestro ambiente?

Muchas de las estimaciones a estos niveles se realizan mediante técnicas matemáticas de extrapolación numérica. Usan censos o estadísticas vitales, información poco confiable en unidades pequeñas. También, en la medida que los cálculos se alejan del punto de partida los resultados suelen ser muy diferentes a la realidad. Por eso es que la utilización de 
técnicas para determinar los indicadores demográficos a niveles pequeños basados en tendencias anteriores, son muy limitados. Es querer pronosticar la frecuencia relativa cuando hay pocos datos, cualquier cambio modifica la frecuencia. La ley de los grandes números apoya este argumento.

Son pocas las proyecciones que se han elaborado a niveles de mayor desagregación geográfica. Este trabajo lo ha cubierto principalmente el Consejo Nacional de Población, debido a que los organismos de planeación estatal requieren de información demográfica por municipio para hacer su trabajo. Habría que fortalecer a los organismos de planeación en los estados para que dispongan de especialistas que hagan sus propias proyecciones. Esto se ha intentado desde hace muchos años pero los cambios políticos estatales no permiten continuidad en la formación de especialistas en demografía. Quienes habitan esas zonas son quienes las deberían hacer porque conocen mejor la realidad social y demográfica de su estado.

Generalmente el método de proyección que se usa es el de los componentes desarrollado por Patrick Holt Leslie a mitad del siglo XX. Los indicadores de la fecundidad y la mortalidad se ubican en una matriz, lo que permite aprovechar el instrumental conceptual del Algebra Lineal. La metodología es muy robusta, aunque proyecta separadamente cada uno de los componentes demográficos, por lo que no considera la interferencia entre ellos. Se proyecta el número de supervivientes de un momento a otro con probabilidades de sobrevivencia y se estiman los nacimientos futuros a partir de las tasas globales de fecundidad proyectadas.

En los años setenta del siglo pasado se tenía un sistema llamado Geomunicipal que contaba con información sociodemográfica precisamente a nivel municipal. La población se estimaba para años futuros a partir de la tasa de crecimiento de la población calculada según los últimos dos censos. Se suponía que la tasa de crecimiento demográfico era constante y se utilizaba como método de pronóstico la función exponencial, la geométrica o la lineal. A veces también la logística. Estos resultados servían para analizar el escenario que se presentaría en el caso de que no cambiarán en el tiempo los niveles de los componentes demográficos: natalidad, mortalidad y migración.

Es importante señalar que en la medida que se estima y se proyecta la población de unidades geográficas más pequeñas, las estimaciones y proyecciones de los indicadores de población son más volátiles. Esto está íntimamente ligado, como ya se decía, a la Ley de los Grandes Números. Si lanzamos una moneda diez veces, es posible que puedan salir más águilas que soles, pero si la lanzamos un millón de veces, el porcentaje de soles 
y de águilas será prácticamente igual, desde luego si la moneda está bien hecha.

Además, los datos sociales y demográficos a esos niveles pequeños son escasos y los que existen están sub o sobre estimados. Cualquier cambio en el numerador o en el denominador de los indicadores de razón produce grandes variaciones en él. Se han hecho pocos esfuerzos por desarrollar métodos de estimación y pronóstico más certeros a esos niveles. Existen dos razones fundamentales, una es que no se dispone de información demográfica a niveles micro-regionales y la otra es que cuando existen estimaciones a esos niveles, las bandas de confianza son muy amplias, cuando se pueden medir, debido a que se cuenta con pocos datos y a que están sujetas a una gran variabilidad. Casi nadie realiza estimaciones demográficas a esos niveles y los que sí las hacen son muy atrevidos por utilizar sólo censos y estadísticas vitales, y métodos de proyección muy simples, aplicados mecánicamente. Su uso es poco útil. Hay personas que toman estos datos de proyección al pie de la letra, sin pensar en su calidad, como si hubieran sido estimados y proyectados por científicos sociales que se aproximan más a un futurólogo o por adivinadores que usando su bola de cristal siempre intentan atisbar el futuro. En nuestro país hace falta una mayor cultura matemática y aritmética, y sobre todo una mente más analítica y reflexiva.

Cuando se realizan pronósticos suponiendo que los componentes demográficos son constantes, su utilidad tiene que ver con responder a la pregunta: ¿qué pasaría si el crecimiento permaneciera constante? Estas proyecciones son muy útiles porque es un punto de comparación contra una proyección meta que se haga cambiando la trayectoria de las tasas de crecimiento demográfico. La diferencia entre ambas nos mide el esfuerzo que se tiene que hacer para llegar a ese fin.

\section{Propósito}

El objetivo de este artículo es proponer una metodología que permita calcular la población de la localidad de Tenosique, Tabasco, México combinando diferentes fuentes demográficas, por un lado los censos de población y las estadísticas vitales, y por el otro, a partir de imágenes satelitales, de ahí el título del trabajo: Estimaciones de la población desde el cielo como desde la tierra. El método empleado es el Filtro de Kalman. El Filtro tiene dos ecuaciones, una de transición y otra de observación que interactúan. Ambas formas de estimación dan resultados diferentes. Cada uno de los resultados se compara en forma a posteriori con el dato "real" del censo. Se ve cual es el mejor en función del resultado de la población y de la va- 
rianza. Se calculan los ponderadores, la mejor estimación tendrá un valor de ponderación más alto. Los dos ponderadores deben sumar uno.

Se ha escogido Tenosique para este ejercicio con el fin de estudiar el efecto del crecimiento poblacional sobre la zona de influencia del rio Usumacinta, derivado de la importante población flotante de migrantes centroamericanos. Hay que reconocer de antemano que desde el espacio es difícil apreciar a la población en fuerte movimiento, pero puede darnos pistas de la movilidad de la población migrante en esta zona de estudio.

Es importante señalar que este estudio forma parte de la siguiente investigación: "Potenciar la resiliencia de las ciudades y sus territorios de pertenencia en el marco de los acuerdos sobre cambio climático y de la Nueva Agenda Urbana" ejecutado por la CEPAL, en colaboración con la cooperación francesa, y el programa EUROCLIMA+, con financiamiento de la Unión Europea y participación del CentroGeo (Rodríguez Aldabe, 2018).

Desde el punto de vista demográfico también resulta interesante porque se trata de una región que presenta fuertes flujos migratorios y se prevé que crecerá en los próximos años debido a los cambios en las rutas migratorias. Además, el municipio presenta un patrón de dispersión de su población según el cual poco más de la mitad de los individuos se ubica en localidades rurales, lo que representa dificultades obvias de estimación de su población con base en los censos; adicionalmente, representa un reto técnico para su mapeo a partir de la percepción remota.

\section{DifiCULTADES Y NECESIDAD DE DATOS PARA LA ESTIMACIÓN DE INFORMACIÓN DEMOGRÁFICA EN ÁREAS PEQUEÑAS}

Es importante destacar que en las áreas pequeñas se dispone de la población total, y en raras ocasiones se cuenta con información por grandes grupos de edades. Tampoco se cuenta en estos niveles con datos sobre los componentes de la dinámica demográfica: mortalidad, fecundidad y mucho menos con datos de la migración interna e internacional. Cuando se tiene información a este nivel, un pequeño aumento o disminución en el valor del numerador o del denominador de las tasas demográficas, produce un cambio muy significativo en los componentes de la dinámica demográfica. Los resultados de los índices son muy sensibles a cambios en el número de los eventos demográficos. En la medida que los pronósticos se alejan del momento censal, estos métodos pueden arrojar resultados muy diferentes de la realidad. 
En los últimos años se ha incrementado la demanda de datos sociodemográficos a nivel micro regional. Cada vez más es necesario contar con mejores estimaciones a nivel de área menor, debido al interés que han despertado estas comunidades para la realización de planes y programas de desarrollo local. Por ejemplo, en países como México existe un enorme número de localidades pequeñas. Según el censo levantado en 2010 hay 189 mil localidades de menos de 2,500 habitantes, donde habita 23 por ciento de la población del país. Muchas de ellas son además las áreas más pobres del país, donde habita un número importante de población indígena, aislada y dispersa, a la que es difícil dirigir programas para mejorar su calidad de vida. Este patrón de dispersión y aislamiento constituye uno de los retos más desafiantes de la planeación social y demográfica. Estas comunidades y su población experimentan los más intensos rezagos sociodemográficos junto con la pobreza extrema y la marginación. Por tal motivo, es importante disponer de información demográfica con el fin de impulsar intervenciones públicas más eficaces para revertir la situación de desventaja en la que se encuentran millones de familias. El interés por disponer de datos a nivel micro regional ha sido impulsado por los gobiernos nacionales y locales, con el fin de focalizar acciones de programas sociales. Frente a esta situación, es necesario contar con información a nivel de áreas pequeñas, por lo que las instituciones generadoras de estadísticas promueven la producción de este tipo de datos, así como por la necesidad de contar con técnicas específicas para estimar información de áreas geográficas menores. Las políticas destinadas a atender las necesidades básicas de la población dan mayor responsabilidad a los gobiernos locales, lo que se ha manifestado en una mayor demanda de información a ese nivel de análisis. Por consiguiente, cada vez crece más la preocupación por tener estimaciones de datos demográficos a nivel más pequeño. Se requiere información sobre la población total, pero también por grupos de edades y sexo y según variables socioeconómicas.

Las proyecciones y las estimaciones de la población a nivel nacional y estatal, como ya se dijo, se han elaborado a partir del método de los componentes, calculando los niveles de la fecundidad, de la mortalidad y de la migración interna e internacional. Esta metodología ha sido posible reproducirla de manera limitada en áreas pequeñas debido a que es difícilmente aplicable por la falta de la información, pero sobre todo a que los indicadores presentan un elevado nivel de variabilidad. Por eso generalmente estas estimaciones a niveles de áreas menores se llevan a cabo mediante el uso de métodos matemáticos, los cuales tienen una utilidad muy restringida, ya 
que son cálculos realizados mecánicamente que no se ajustan a la realidad y sobre todo no es posible medir su nivel de confiabilidad.

Una ventaja que se tiene al realizar estimaciones de población con base en los datos vía satélite, es que se dispone de datos virtualmente en tiempo real, por lo que se pueden hacer ajustes de la población en forma continua. Además, desde el espacio también es posible hacer un análisis de las desigualdades, de la pobreza y de la marginación tomando en cuenta los lugares habitacionales vistos desde el cielo.

\section{Demografía de Tenosique}

¿Qué pasa en la demografía de Tenosique? Su crecimiento poblacional es lento, es un poco menor a uno por ciento. Se sabe que hay una fuerte migración de indocumentados desde Honduras, El Salvador y Guatemala. Salen huyendo de la violencia y la pobreza. "Según ACNUR (Agencia de la ONU para Refugiados), en 2016 México recibió cerca de nueve mil nuevas solicitudes de asilo, un aumento de 156 por ciento en comparación con 2015." (Nájera, 2017). Esta movilidad de población es una migración en tránsito hacia los Estados Unidos. Los migrantes llegan a México en donde pasan unos días, y pronto buscan la vía del tren en espera de que "La Bestia" se aparezca a fin de continuar su viaje hacia el norte. En la actualidad este fenómeno se ha acentuado lo cual se constata con las caravanas de migrantes.

La localidad de Tenosique es pequeña con apenas 32,579 habitantes en el año 2010 y con una superficie en 2015-2016 de 509.1 hectáreas. Muchas de las viviendas son de un piso, por lo que la expansión del área construida nos da una idea del crecimiento de la población. (Ver Tabla 1 y Figura 1). Es posible señalar que existe una alta correlación entre el área y la población. El coeficiente de determinación es elevado e igual a 0.87 . Las viviendas de dos pisos se localizan en el centro de la localidad.

Entre 1990 y 2010 la población de Tenosique aumentó en 9,017 habitantes. La tasa de crecimiento de la población del periodo fue de 1.6 por ciento anual. Mientras que la tasa de crecimiento demográfico de la localidad de Tenosique entre 2005 y 2010 se encuentra por debajo de uno por ciento anual, fue de 0.7 por ciento. De seguir esta tasa de aumento la población se duplicaría cada siglo. Esto muestra una declinación importante en el crecimiento de la población. Hace dos décadas era de 4.3 por ciento (Tabla 1). Si Tenosique hubiera seguido creciendo a 4.3 por ciento anual, la población se hubiera duplicado en lapsos de 16 años. La población sería de 67,502 habitantes en el 2015, cifra igual al doble de la censada en 2010. 
Tabla 1: Ciudad de Tenosique: Población, área, tasa de crecimiento de la población y del área

\begin{tabular}{rrrrr}
\hline Año & Población & $\begin{array}{r}\text { Área } \\
(\text { ha })\end{array}$ & $\begin{array}{r}\text { Tasa de crecimiento } \\
\text { de la población }\end{array}$ & $\begin{array}{r}\text { Tasa de crecimiento } \\
\text { del área }\end{array}$ \\
\hline 1991 & $23,562(1)$ & 269.3 & $4.3 \%$ & $4.6 \%$ \\
1996 & $29,134(2)$ & 338.0 & $0.6 \%$ & $5.6 \%$ \\
2001 & $30,042(3)$ & 443.9 & $0.9 \%$ & $1.1 \%$ \\
2006 & $31,392(4)$ & 468.9 & $0.7 \%$ & $1.0 \%$ \\
2011 & $32,579(5)$ & 492.0 & & $0.7 \%$ \\
\hline
\end{tabular}

Nota: Cálculo de área sobre pixeles en la proyección UTM Z15.

Fuente:

(1) Censo de Población de 1990. Población referida a 1990.

(2) Conteo de Población de 1995. Población referida a 1995.

(3) Censo de Población de 2000. Población referida a 2000.

(4) Conteo de Población de 2005. Población referida a 2005.

(5) Censo de Población de 2010. Población referida a 2010.

Figura 1: Tenosique: Población y área, 1990-2010

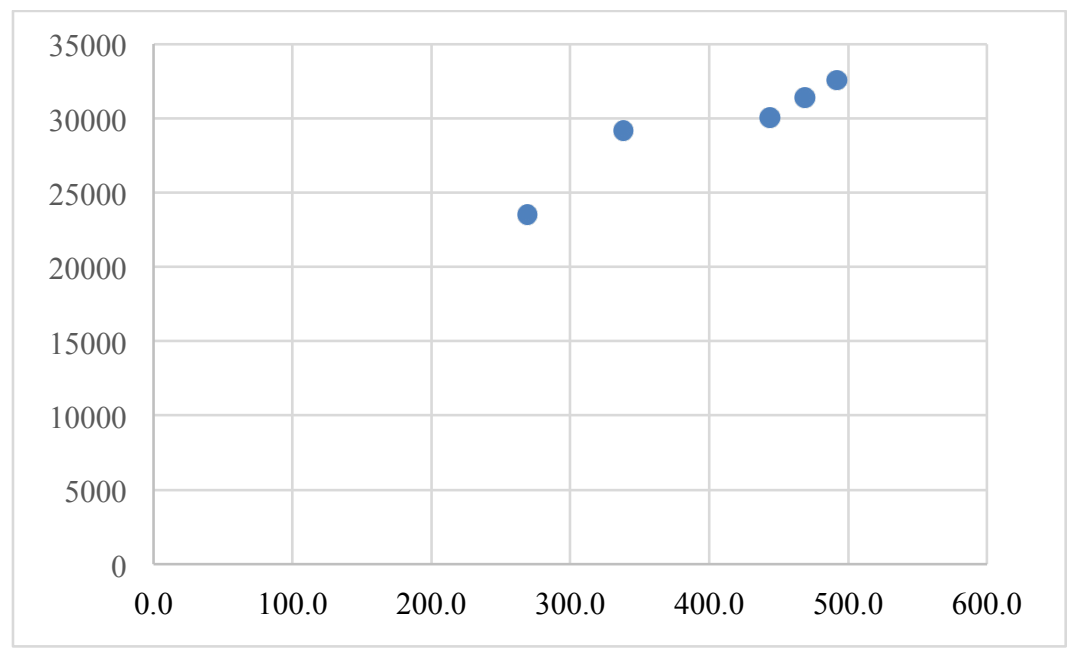

Los cálculos de las áreas son elaboraciones propias sobre pixeles en la proyección UTM Z15.

Fuente: INEGI. Censos de población de 1990, 2000, y 2010 y Conteos de población de 1995 y 2005.

Sin embargo si uno observa el crecimiento de la población de las últimas décadas se aprecia un estancamiento de la población con pequeños aumentos. De 1995 a 2010 la población sólo aumentó un poco más de tres mil personas.

Estos cambios en el ritmo de crecimiento demográfico del municipio de Tenosique obedecen al hecho de que la fecundidad ha descendido rápido. El número promedio de hijos de las mujeres de 45 a 49 años según el censo 
de 2010 fue de 3.8; el del total del estado fue de 3.63 y a nivel nacional el censo dio 3.33. Las mujeres al final de su vida fértil nacidas en Tenosique en la primera mitad de los años cuarenta del siglo pasado, tenían 6.27 hijos por mujer en promedio. Casi el doble de lo que se observó en 2010, lo que muestra una reducción acelerada en los niveles de fecundidad.

Los niveles de mortalidad en el municipio de Tenosique también han descendido aceleradamente. El porcentaje de hijos fallecidos de las mujeres de 45 a 49 años fue de 5.5 por ciento y el de las mujeres de 65 a 69 y 70 a 74 años fue de 13.17 por ciento y 14.07 por ciento, respectivamente. En el estado de Tabasco dicho porcentaje fue de 6.12 por ciento para las mujeres de 45 a 49 años y de 13.62 por ciento y de 15.76 por ciento, para las mujeres de 65 a 69 y 70 a 74 años, respectivamente. Como se puede observar, dichos porcentajes son más bajos en Tenosique que en el estado. Estas cifras son de sólo 3.8 por ciento para las mujeres de 45 a 49 años, de 8.77 por ciento para las de 65 a 69 años y de 10.86 por ciento para las de 70 a 74 años.

Al analizar la información sobre migración hacia el municipio de Tenosique, tomando en cuenta la residencia anterior hace cinco años se encuentra que Tenosique en 2010 recibió población principalmente de Chiapas (505), Quintana Roo (309) y Campeche (161), en ese orden de importancia numérica. Llama la atención que después de Chiapas y Quintana Roo el municipio recibió población de Estados Unidos (265). Hay 64 migrantes que provienen de otro país y un número elevado de no especificados (163) que pudieran haber migrado desde alguno de los países centroamericanos que están más vinculados económicamente con México.

Es importante destacar que la ciudad de Tenosique representa alrededor de la mitad de la población del municipio. Dicha proporción se ha mantenido prácticamente constante a lo largo de dos decenios. Esto significa que el crecimiento de la localidad no ha sido muy distinto del observado en el resto del municipio (Tabla 2).

\section{Información disponible}

Las fuentes de información con las que se cuenta para realizar este trabajo son los censos de 1990, 2000 y 2010 y los Conteos de Población de 1995 y 2005 e imágenes satelitales de la superficie para los años de 1991, 1996, 2001, 2006, 2011 y 2016, medida en hectáreas. 
Tabla 2: Población de la localidad de Tenosique, del municipio y proporción de la población de la localidad respecto a la municipal, 1990-2010

\begin{tabular}{rrrr}
\hline Año & Localidad & Municipio & Proporción \\
\hline 1990 & 23,562 & 47,642 & 0.49 \\
1995 & 29,134 & 55,438 & 0.53 \\
2000 & 30,042 & 55,712 & 0.54 \\
2005 & 31,392 & 55,601 & 0.56 \\
2010 & 32,579 & 58,960 & 0.55 \\
\hline
\end{tabular}

Fuente: Censos y Conteos de Población.

\section{Cuantificación del área urbana de Tenosique}

Un camino alterno para analizar la dinámica demográfica de Tenosique es a través de las imágenes de la zona desde el espacio. La región del sitio de estudio se encuentra en una zona tropical que permanece cubierta por nubes gran parte del año por lo que resulta un tanto difícil su observación a partir de sensores ópticos a bordo de los satélites de monitoreo de la tierra. No obstante, el acervo de imágenes más antiguas que se tienen hoy día lo constituyen precisamente las imágenes captadas con sensores ópticos. En ese sentido, para la cuantificación de las áreas urbanas se emplearon imágenes satelitales de las series Landsat 5 y Landsat 7 (Loveland y Dwyer, 2012) disponibles en forma gratuita por el Servicio Geológico de los Estados Unidos (USGS por sus siglas en inglés). Aunque las imágenes han sido procesadas para representar la fracción de luz reflejada (reflectancia) de la superficie terrestre, en esta región no es posible eliminar por completo los efectos atmosféricos y en muchos casos la información presenta huecos debido a la nubosidad densa. Afortunadamente, se cuenta con varias tomas al año (Tabla 3), y considerando que el área representada por un pixel de la imagen puede ser visible desde el espacio en algún momento del año, es posible generar una imagen prácticamente libre de nubes y huecos a partir del estadístico de mediana sobre las imágenes del mismo año, es decir, se forma una imagen a partir de múltiples imágenes tomadas en el mismo año, donde el valor del pixel seleccionado es la mediana de los valores de las distintas imágenes. La imagen resultante excluye los valores que corresponden a las nubes (blancos) o a las sombras (negros) porque estos valores se encuentran en los extremos del rango. 
Tabla 3: Número de escenas Landsat por año, seleccionadas para formar el compuesto anual

\begin{tabular}{lcc}
\hline Año & Sensor & Imágenes \\
\hline 1984 & LT5 & 1 \\
1991 & LT5 & 7 \\
1996 & LT5 & 13 \\
2001 & LT5 & 8 \\
2006 & LE7 & 15 \\
2011 & LT5 & 5 \\
2015 & LE7 & 18 \\
\hline
\end{tabular}

Fuente: elaboración propia a partir de la información del Servicio Geológico de los Estados Unidos (USGS).

Las imágenes libres de nubes fueron sometidas a un procesamiento de demezclado espectral de acuerdo con el cual se modelan los pixeles de la imagen como la mezcla de materiales puros distribuidos horizontalmente en toda el área (Silván-Cárdenas y Wang, 2010). El demezclado espectral permite cuantificar la abundancia o cobertura relativa de los materiales puros, tales como superficies impermeables, suelo desnudo, vegetación y agua. En particular, la fracción de superficie impermeable determinada con este método (Figura 2) fue empleada para estimar el área del asentamiento en varios años. Para este fin, el área urbana (Tabla 4) se definió como el área de los pixeles que presentaron 25 por ciento de cobertura de superficie impermeable o mayor, de tal forma que el total del área cubierta por los pixeles que cumplen éste criterio y que además se encuentran dentro del área geoestadística de los datos censales, constituye el valor de área urbana estimado.

La idea de estimar la población a partir del área urbana de los asentamientos, donde el área urbana se define en términos de superficies impermeables detectadas por satélite ( $\mathrm{Lu}$ y Li, 2006), es casi tan antigua como los propios satélites. Alrededor del mundo existen numerosos estudios que dan cuenta de la viabilidad de este enfoque considerando distintas definiciones del espacio habitado (Silván-Cárdenas y otros, 2015). Este estudio pretende dar un paso más allá al considerar la dinámica de ambas variables: Población y Área Urbana, en un periodo razonable de tiempo.

Se han realizado dos estimaciones del área: la primera incluye toda el área de la localidad de Tenosique, la segunda considera sólo la superficie construida. Ambas están estrechamente relacionadas por lo que proporcionan estimaciones semejantes debido a que existe un alto coeficiente de determinación entre ambas de 0.93 , lo que significa que la parte explicada por la regresión es 93 por ciento y la explicada por los residuos es sólo de siete por ciento (Tabla 5 y Figura 3). 
Estimaciones de las población desde la tierra como desde el cielo: el caso de Tenosique /M. ORDORICA et al.

Figura 2: Fracción de cobertura urbana sobrepuesta en las imágenes de mediana
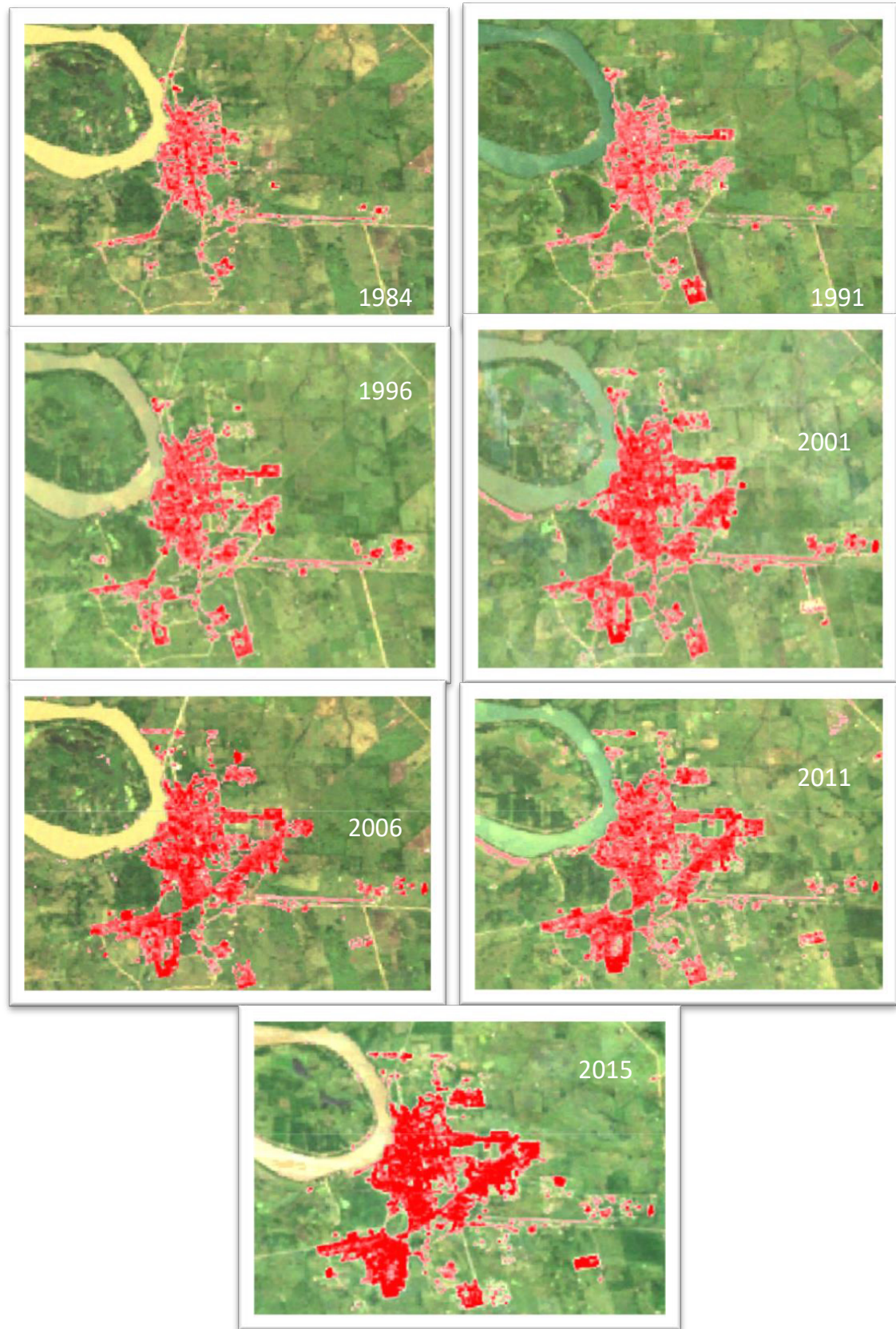

Fuente: elaboración propia a partir de imágenes de la serie de satélites Landsat 5 y 7 del Servicio Geológico de los Estados Unidos (USGS). 
Tabla 4: Superficie urbana y superficie impermeable por densidad de cobertura Área impermeable por densidad de cobertura (ha)

\begin{tabular}{lrrrrr}
\hline Año & $\begin{array}{r}\text { Área urbana } \\
\text { (ha) }\end{array}$ & $\begin{array}{r}\text { Baja } \\
(25-50 \%)\end{array}$ & $\begin{array}{r}\text { Media } \\
(50-75 \%)\end{array}$ & $\begin{array}{r}\text { Alta } \\
(75-100 \%)\end{array}$ & Total \\
\hline 1984 & 194.2 & 28.5 & 62.6 & 19.6 & 110.8 \\
1991 & 255.7 & 64.4 & 76.1 & 17.4 & 157.8 \\
1996 & 320.7 & 42.0 & 129.1 & 42.5 & 213.6 \\
2001 & 306.6 & 51.4 & 149.7 & 93.1 & 294.1 \\
2006 & 445.0 & 46.2 & 142.9 & 134.1 & 323.2 \\
2011 & 466.6 & 61.6 & 180.2 & 101.6 & 343.4 \\
2015 & 482.0 & 26.0 & 77.4 & 263.5 & 366.9 \\
\hline
\end{tabular}

Fuente: elaboración propia.

Tabla 5: Tenosique: áreas 1 y 2

\begin{tabular}{lrr}
\hline & Área 1 & Área 2 \\
\hline 1984 & 204.9 & 111.3 \\
1991 & 269.3 & 134.5 \\
1996 & 338 & 193.9 \\
2001 & 443.9 & 267.9 \\
2006 & 468.9 & 302.1 \\
2011 & 492 & 297.7 \\
2015 & 509.1 & 389.5 \\
\hline Nota: El área 1 corresponde al total de la superficie de Tenosique y el área 2 corresponde al \\
área construida. \\
Fuente: elaboración propia.
\end{tabular}

\section{El Filtro de Kalman}

Se puede decir que el Filtro de Kalman es una versión moderna del método de los Mínimos Cuadrados. Existe una diferencia esencial entre ambas técnicas. En el método de los Mínimos Cuadrados los parámetros son variables aleatorias, es decir son fijos, y en el Filtro de Kalman los parámetros son procesos estocásticos, esto quiere decir que cambian con el tiempo. La idea de filtro es similar a lo que uno hace cuando se elimina alguna impureza del agua o se reduce el ruido cuando se está sintonizando una estación de radio. Es importante destacar que así como ocurrió con el método de los Mínimos Cuadrados sobre la disputa Legendre-Gauss sobre quien fue 
el creador del método, algo similar ocurrió entre Swerling-Kalman sobre quien fue el pionero en la aplicación del método. Mientras en la controversia de quien fue el creador de la técnica de los Mínimos Cuadrados entre Gauss y Legendre fue analizada y discutida en el ámbito académico, la controversia Kalman-Swerling no ha trascendido. En 1960 en ocasión de la visita de Kalman a la National Aeronautic and Space Administration (NASA) se analizó el potencial de utilización de la técnica a la estimación de la trayectoria del Apolo a la Luna. El Filtro tiene numerosas aplicaciones y en este artículo se intenta hacer una aplicación al campo de las estimaciones poblacionales en el tiempo, vistas también como una trayectoria.

Figura 3: Tenosique: Superficie (en hectáreas) del total de la zona, ubicada en el eje X versus área habitada (en hectáreas) ubicada en el eje Y para los años 1984, 1991, 1996, 2001, 2006, 2011 у 2015

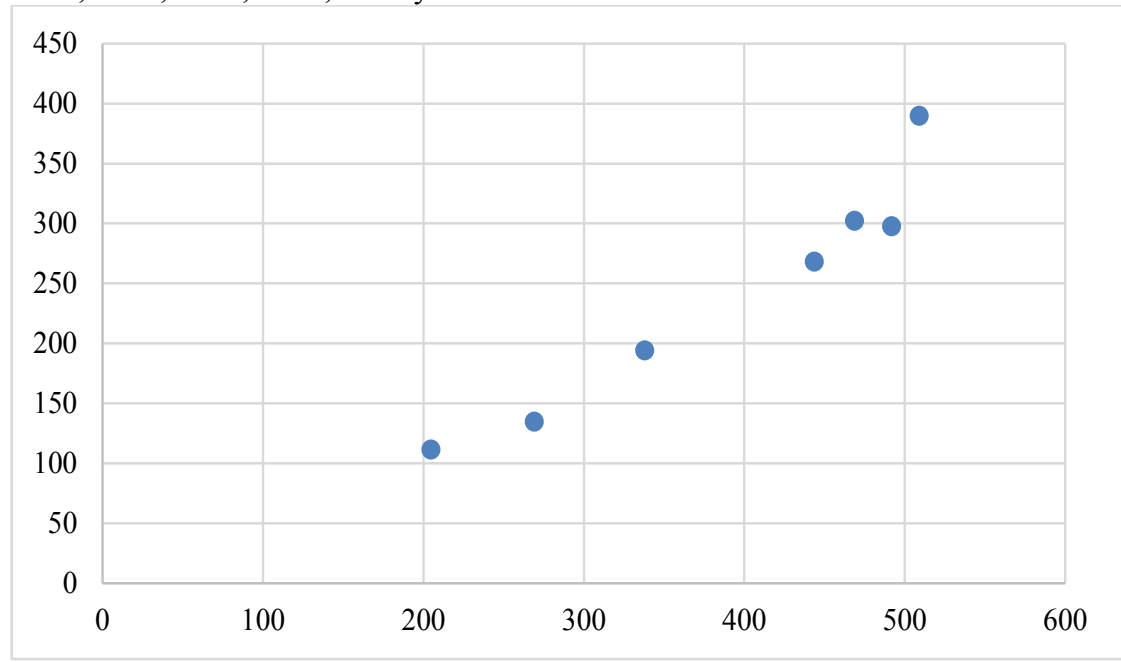

Fuente: elaboración propia a partir de la Tabla 5 .

Existen caminos alternos para encontrar previsiones a nivel local a través de métodos basados en variables sintomáticas. Estas variables se definen como un conjunto de datos que se encuentran relacionados con los cambios en el tamaño de la población. Se trata de información de registros administrativos actualizados para áreas pequeñas, la cual se relaciona con las variables ligadas a la dinámica demográfica. Esta información sirve para hacer estimaciones sobre datos más actuales hasta que se realiza un nuevo censo. Las variables sintomáticas deben presentar una elevada asociación con el número y la dinámica de la población, y deben ser registros continuos, tales como las estadísticas vitales y la matrícula escolar, entre 
otras. La relación puede no ser perfecta pero permite aproximarnos a la realidad si la variable fue bien elegida.

El método que se propone en este trabajo cae en esta categoría, en la medida en que se utiliza información continua pero desde el espacio. El satélite pasa por el mismo lugar en forma frecuente. El método consiste en establecer una relación entre el crecimiento del área y el incremento de su población. Para realizar este trabajo se ha seleccionado el Filtro de Kalman, el cual está formado por dos ecuaciones:

$\mathrm{X}_{\mathrm{K}+1}=\Phi_{\mathrm{K}} \mathrm{X}_{\mathrm{K}}+\mathrm{W}_{\mathrm{K}}$, llamada ecuación de transición y $\mathrm{Z}_{\mathrm{K}}=\mathrm{H}_{\mathrm{K}} \mathrm{X}_{\mathrm{K}}+\mathrm{V}_{\mathrm{K}}$, llamada ecuación de observación.

Una variante del modelo anterior es la siguiente:

$$
\mathrm{X}_{\mathrm{K}+1}^{\prime}=\Phi_{\mathrm{K}} \mathrm{X}{ }_{\mathrm{K}, \varnothing}+\mathrm{W}_{\mathrm{K}} \mathrm{y} \mathrm{X}^{\prime}{ }_{\mathrm{K}+1}=\mathrm{r}_{\mathrm{K}, \mathrm{S}} \mathrm{X}{ }_{\mathrm{K}, \mathrm{S}}+\mathrm{V}_{\mathrm{K}}
$$

En la primera ecuación $X^{\prime}{ }_{K+1}$ es la población en el momento $\mathrm{K}+1$ estimada a partir de la tasa de crecimiento poblacional, $\Phi_{\mathrm{K}}$ es la tasa de crecimiento demográfico entre $\mathrm{K}$ y $\mathrm{K}+1, \mathrm{X}_{\mathrm{K}, \varnothing}$, es la población en el instante $\mathrm{K}$.

En la segunda ecuación $X{ }^{\prime}{ }_{\mathrm{K}+1}$ es la población en el momento $\mathrm{K}+1$ estimada a partir de la tasa de crecimiento de la superficie, $\mathrm{r}_{\mathrm{K}, \mathrm{S}}$ es la tasa de crecimiento de la superficie entre $\mathrm{K}$ y $\mathrm{K}+1, \mathrm{X}{ }{ }_{\mathrm{K}, \mathrm{S}}$ es la población en $\mathrm{K}, \mathrm{W}_{\mathrm{K}}$ $\mathrm{y} \mathrm{V}_{\mathrm{K}}$ son errores aleatorios. Al inicio $\mathrm{X}_{\mathrm{K}, \varnothing} \mathrm{y} \mathrm{X}^{\prime \prime, ~}{ }_{\mathrm{K}, \mathrm{S}}$ coinciden.

La estimación final de la población combina el cálculo con base en el crecimiento demográfico con el obtenido a partir del incremento de la superficie. Es una suma ponderada de las dos:

$$
\mathrm{X}_{\mathrm{Kest}}=\mathrm{r}_{\mathrm{K}}\left(\mathrm{X}_{\mathrm{K}, \varnothing}\right)+\left(1-\mathrm{r}_{\mathrm{K}}\right)\left(\mathrm{X}_{\mathrm{K}, \mathrm{S}}\right) \text {. }
$$

Al final, en la Figura 4 y la Tabla 6 se presentan los resultados de las estimaciones y proyecciones de población de Tenosique hasta el año 2020 usando el Filtro de Kalman.

\section{CONCLUSIÓN Y DISCUSIÓN FINAL}

El método es útil para estimar la población prácticamente en tiempo real, el satélite permite observar continuamente el área de interés, sin embargo, después de analizar las tablas y mapas es posible destacar que la ciudad de Tenosique ha crecido muy lentamente entre el año 2000 y el 2015, lo que se observa a partir de la información censal y también con base en el crecimiento del área. Hubiéramos esperado un incremento de la población mayor debido a la importante inmigración de centroamericanos. La población fue censada mediante el método de jure o de derecho, es decir, se captan a 
los residentes habituales. El método no cuenta a la población flotante que vive en albergues por periodos muy breves.

Figura 4: Tenosique: Población estimada con la tasa de crecimiento demográfico $\left(\mathrm{X}_{\mathrm{k}, \varnothing}^{\prime}\right)$, población estimada con el área $\left(\mathrm{X}^{\prime \prime}{ }_{\mathrm{k}, \mathrm{S}}\right)$, población final ponderada $\left(\mathrm{X}_{\text {kest }}\right)$

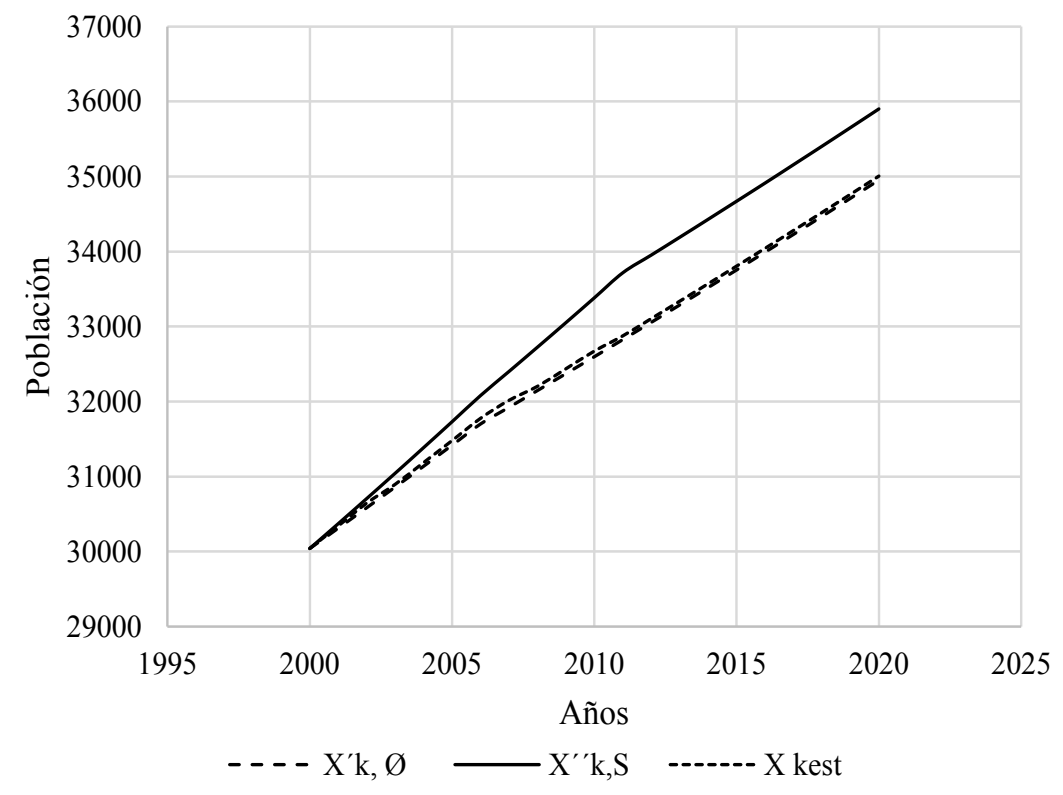

Fuente: elaboración propia a partir de la Tabla 6.

La superficie de la ciudad de Tenosique no aumenta, pero los migrantes que llegan y se van son numerosos. Habría que incorporar algún otro elemento que dé cuenta de su paso por Tenosique, por ejemplo, consumo de alimentos y de líquidos, entre otros. Este método puede ser muy útil para estimar la población tomando en cuenta no sólo el área sino también el volumen de la región de análisis, es decir, la altura de casas y edificios. 
Tabla 6: Ciudad de Tenosique: tasas de crecimiento demográfico $\left(\emptyset_{\mathrm{k}}\right)$ y del área $\left(\mathrm{r}_{\mathrm{ks}}\right)$; población estimada a partir de la tasa de crecimiento demográfico $\left(X_{\mathrm{k}, \emptyset}, \mathrm{y}\right.$ y del área $\left(X_{\mathrm{k}, \mathrm{S}}{ }^{\prime}\right) ; \mathrm{H}_{\mathrm{k}}$ es el valor del área; población final estimada en forma ponderada de acuerdo a las dos estimaciones anteriores $\left(X_{\text {kest }}\right)$; varianzas de los errores aleatorios $\left(\sigma_{\emptyset}^{2}\right.$ y $\left.\sigma_{S}^{2}\right)$; suma de varianzas $\left(\sigma_{\emptyset}^{2}+\sigma_{S}^{2}\right)$, ponderación $(\rho), 2000-2020$

\begin{tabular}{|c|c|c|c|c|c|c|c|c|c|c|c|}
\hline Año & $\emptyset_{k}$ & $X_{k, \emptyset}^{\prime}$ & $\sigma_{\emptyset}^{2}$ & $\mathrm{H}_{\mathrm{k}}$ & $\mathrm{r}_{\mathrm{kS}}$ & $X{ }_{k, S}^{\prime}$ & $\left.\left(X^{\prime \prime} / X^{\prime}\right)-1\right)$ & $\sigma_{S}^{2}$ & $\sigma_{\emptyset}^{2}+\sigma_{S}^{2}$ & $\rho$ & $X_{\text {kest }}$ \\
\hline 2000 & 1.009 & 30042 & 22563 & 438.9 & 1.011 & 30042 & 0.00 & 22563 & 45126 & 0.50 & 30042 \\
\hline 2001 & 1.009 & 30312 & 22971 & 443.9 & 1.011 & 30372 & 0.00 & 23062 & 46033 & 0.50 & 30342 \\
\hline 2002 & 1.009 & 30585 & 23386 & 448.9 & 1.011 & 30707 & 0.00 & 23572 & 46959 & 0.50 & 30646 \\
\hline 2003 & 1.009 & 30860 & 23809 & 453.9 & 1.011 & 31044 & 0.01 & 96375 & 120184 & 0.20 & 30897 \\
\hline 2004 & 1.009 & 31138 & 24240 & 458.9 & 1.011 & 31386 & 0.01 & 98507 & 122747 & 0.20 & 31187 \\
\hline 2005 & 1.009 & 31418 & 24678 & 463.9 & 1.011 & 31731 & 0.01 & 100686 & 125364 & 0.20 & 31480 \\
\hline 2006 & 1.007 & 31701 & 25124 & 468.9 & 1.010 & 32080 & 0.01 & 102913 & 128037 & 0.20 & 31776 \\
\hline 2007 & 1.007 & 31923 & 25477 & 473.5 & 1.010 & 32401 & 0.01 & 104982 & 130459 & 0.20 & 32016 \\
\hline 2008 & 1.007 & 32147 & 25835 & 478.1 & 1.010 & 32725 & 0.02 & 240957 & 266792 & 0.10 & 32203 \\
\hline 2009 & 1.007 & 32372 & 26198 & 482.8 & 1.010 & 33052 & 0.02 & 245800 & 271998 & 0.10 & 32437 \\
\hline 2010 & 1.007 & 32598 & 26566 & 487.4 & 1.010 & 33383 & 0.02 & 250741 & 277307 & 0.10 & 32673 \\
\hline 2011 & 1.007 & 32826 & 26939 & 492.0 & 1.007 & 33717 & 0.03 & 454721 & 481661 & 0.06 & 32876 \\
\hline 2012 & 1.007 & 33056 & 27318 & 496.3 & 1.007 & 33953 & 0.03 & 461110 & 488427 & 0.06 & 33106 \\
\hline 2013 & 1.007 & 33288 & 27702 & 500.6 & 1.007 & 34190 & 0.03 & 467588 & 495289 & 0.06 & 33338 \\
\hline 2014 & 1.007 & 33521 & 28091 & 504.9 & 1.007 & 34430 & 0.07 & 474157 & 502248 & 0.06 & 33571 \\
\hline 2015 & 1.007 & 33755 & 28485 & 509.1 & 1.007 & 34671 & 0.03 & 480818 & 509304 & 0.06 & 33806 \\
\hline 2016 & 1.007 & 33992 & 28886 & & 1.007 & 34913 & 0.03 & 487573 & 516459 & 0.06 & 34043 \\
\hline 2017 & 1.007 & 34229 & 29291 & & 1.007 & 35158 & 0.03 & 494423 & 523715 & 0.06 & 34281 \\
\hline 2018 & 1.007 & 34469 & 29703 & & 1.007 & 35404 & 0.03 & 501369 & 531072 & 0.06 & 34521 \\
\hline 2019 & 1.007 & 34710 & 30120 & & 1.007 & 35652 & 0.03 & 508413 & 538533 & 0.06 & 34763 \\
\hline 2020 & & 34953 & 30543 & & & 35901 & 0.03 & 515556 & 546099 & 0.06 & 35006 \\
\hline
\end{tabular}

Nota: Para el cálculo de la población de 2010 en adelante, con base en la tasa de crecimiento demográfico, se supone que el crecimiento de la población de 2010 en al 2020 se mantiene constante al observado entre 2005 y 2010, tomando en cuenta el Conteo de 2005 y el Censo de 2010. Para la estimación de la población con base en el incremento del área, se supone que la tasa de crecimiento de la superficie es la misma a la observada entre 2010 y 2015. Los valores del área $\left(\mathrm{H}_{\mathrm{k}}\right)$ para puntos intermedios se obtuvieron interpolando linealmente los datos observados entre: 2001 y 2006; de 2006 a 2011 y entre 2011 y 2015. ( $\left.X^{\prime \prime} / X^{\prime}\right)-1$ es la diferencia porcentual entre ambas estimaciones de población.

Fuente: Las estimaciones de población son elaboraciones propias usando el Filtro de Kalman con base en los censos de población del 2000 y del 2010 y del Conteo de población del 2005. Los cálculos de las áreas se realizaron a partir de la Tabla 5. 


\section{REFERENCIAS BIBLIOGRÁFICAS}

Cavenaghi, S., 2012, Estimaciones y proyecciones de población en América Latina: desafios de una agenda pendiente. Serie e-Investigaciones N. ${ }^{\circ} 2 /$ ALAP Editor, Rio de Janeiro, Brasil, p.108-112.

Gil Olmos, J., 2015, "La 72, una casa para migrantes en medio del infierno criminal", en Proceso, Publicación seriada regular, archivo de los números publicados en proceso.com.mx, 25 de noviembre de 2015. Última consulta: 14/12/2017. Disponible en $\mathrm{http}: / / \mathrm{ww}$.proceso.com.mx/421616/la-72-una-casa-para-migrantesen-medio-del-infierno-criminal

Hernández, O., 2017, “Como Godot, esperando el tren que nunca llega”, en Plaza Pública Publicación regular, 10 de octubre de 2017. Última consulta: 17/10/2017. Disponible en https://www.plazapublica.com.gt/content/como-godot-esperandoel-tren-que-nunca-llega

Loveland, T. R. y Dwyer, J.L., 2012, "Landsat: Building a strong future", en Remote Sensing of Environment, 122, 22-29.

Lu, D., Weng, Q. y Li, G., 2006, "Residential population estimation using a remote sensing derived impervious surface approach", en International Journal of Remote Sensing, 27(16), 3553-3570.

Nájera Coronado, M., 2017, Tenosique en su territorio. Violación de derechos humanos: el caso de los inmigrantes. CentroGeo, Centro Público de Investigación de Conacyt, p. 5.

Ordorica, M y García, V.M., 2016, "Estimating the Demographic Dynamic of Small Areas with the Kalman Filter", en Schoen R. (ed.) Dynamic Demographic Analysis. The Springer Series on Demographic Methods and Population Analysis, vol. 39, Springer, Cham, pp.261-271.

Rodriguez Aldabe, Yosu, 2018, Potenciar la resiliencia de las ciudades y sus territorios de pertenencia en el marco de los acuerdos sobre cambio climático y de la Nueva Agenda Urbana, Documentos de Proyectos (LC/TS.2018/91, Santiago, Comisión Económica para América Latina y el Caribe (CEPAL).

Silván-Cárdenas, JL y Wang, L., 2010, "Fully constrained linear spectral unmixing: Analytic solution using fuzzy sets", en IEEE Transactions on Geoscience and Remote Sensing, 48(11), 3992-4002.

Silván-Cárdenas, JL, Montejano Escamilla, JA y Cervantes Salas, MP, 2015, "Percepción remota para la estimación de población en áreas geoestadísticas básicas", en Realidad Datos y Espacios, 6(1):50-71, Revista Internacional de Estadística y Geografía. 


\section{RESUMEN CURRICULAR DE LOS AUTORES}

\section{Manuel Ordorica Mellado}

Estudió la carrera de actuario en la Facultad de Ciencias de la UNAM; demógrafo de El Colegio de México y doctor en Investigación de Operaciones en la UNAM. Realizó una especialidad en Análisis Demográfico en la Agencia de Cooperación Internacional de Japón. Fue jefe de Evaluación Demográfica en la Dirección General de Estadística y director de Estudios de Población en el CONAPO; consultor en Educación en Población en la UNESCO. Coordinó la Maestría en Demografía y el Doctorado en Estudios de Población en el Colegio de México. Fue Director y Secretario General en el mismo Colegio. Formó parte del Consejo Editorial de la revista Population del INED en París. Ha sido miembro del Comité técnico para la Redistritación y para la Evaluación del Padrón Electoral en el INE. Pertenece al Sistema Nacional de Investigadores nivel III. En 1998 recibió el Premio Nacional de Demografía. Actualmente es profesor-investigador de El Colegio de México y trabaja en demografía matemática.

Dirección electrónica: mordori@colmex.mx

\section{José Luis Silvan Cárdenas}

Recibió el grado de Ingeniero en Computación (1998) y el grado de Maestro en Ingeniería Eléctrica (2002) por la Universidad Nacional Autónoma de México (UNAM), y el PhD en Ciencias de Información Geográficas por la Universidad de Texas (Texas State University-San Marcos 2009). De 2008 a 2010 realizó un postdoctorado en la Universidad de Búffalo (State University of New York at Buffalo). Durante su carrera ha recibido varios reconocimientos, incluyendo la medalla Alfonso Caso que es otorgada por la UNAM y el premio Warren Nystrom en 2011 por la Asociación Americana de Geografía. Es autor de más de 50 publicaciones incluyendo artículos en revistas arbitradas capítulos de libros. Sus temas de investigación incluyen extracción de rasgos geográficos mediante imágenes ópticas y escáner láser aerotransportado.

Dirección electrónica: jlsilvan@centrogeo.edu.mx

\section{Juan Manuel Núñez}

Estudió la maestría y el doctorado en Geomática en el CentroGeo. Realizó su licenciatura en Estudios de Ingeniería Topográfica y Geodesia en la UNAM. Ha participado en el desarrollo de proyectos de investigación aplicada y vinculación en relación a temas de Geomática Aplicada al Medio 
Estimaciones de las población dessde la tierra como desde el cielo: el caso de Tenosique /M. ORDORICA et al.

Ambiente y Percepción Remota Urbana. Imparte cursos sobre Geomática, Sistemas de Información Geográfica, Análisis Espacial, Percepción Remota, Geodesia y Cartografía. Es miembro del Sistema Nacional de Investigadores del CONACYT a partir del 2019.

Dirección electrónica: jnunez@centrogeo.edu.mx

Artículo recibido el 10 de abril de 2018 y aprobado el 27 de julio de 2018. 